\title{
On the Estimation of Mutual Information
}

\author{
Nicholas Carrara * ${ }^{*}$ and Jesse Ernst \\ Physics department, University at Albany, 1400 Washington Ave, Albany, NY 12222, USA; jae@albany.edu \\ * Correspondence: ncarrara@albany.edu \\ + Presented at the 39th International Workshop on Bayesian Inference and Maximum Entropy Methods in \\ Science and Engineering, Garching, Germany, 30 June-5 July 2019.
}

Published: 15 January 2020

check for updates

\begin{abstract}
In this paper we focus on the estimation of mutual information from finite samples $(\mathcal{X} \times \mathcal{Y})$. The main concern with estimations of mutual information (MI) is their robustness under the class of transformations for which it remains invariant: i.e., type I (coordinate transformations), III (marginalizations) and special cases of type IV (embeddings, products). Estimators which fail to meet these standards are not robust in their general applicability. Since most machine learning tasks employ transformations which belong to the classes referenced in part I, the mutual information can tell us which transformations are most optimal. There are several classes of estimation methods in the literature, such as non-parametric estimators like the one developed by Kraskov et al., and its improved versions. These estimators are extremely useful, since they rely only on the geometry of the underlying sample, and circumvent estimating the probability distribution itself. We explore the robustness of this family of estimators in the context of our design criteria.
\end{abstract}

Keywords: mutual information; non-parametric entropy estimation; dimension reduction; machine learning

\section{Introduction}

Interpretting mutual information (MI) as a measure of correlation has gained considerable attention over the past couple of decades for it's application in both machine learning [1-4] and in dimensionality reduction [5], although it has a rich history in Communication Theory, especially in applications of Rate-Distortion theory [6] (While MI has been exploited in these examples, it has only recently been derived from first principles [7]). MI has several useful properties, such as having a lower bound of $I_{\mathrm{min}}=0$ for variables which are uncorrelated. Mostly we are interested in how the MI changes whenever we pass one set of variables through a function, which according to the data processing inequality can only ever destroy correlations and not increase them. Thus, the MI for any set of variables $\mathbf{X} \times \mathbf{Y}$ is an upper bound for any transformation $f(\mathbf{X}) \times g(\mathbf{Y})$. This feature makes MI a good measure of performance for any machine learning (ML) task, which is why has gained so much attention recently [1-4].

The main challenge with using $\mathrm{MI}$ in any inference task is computing it when one only has a sample $\mathcal{X} \times \mathcal{Y} \subset \mathbf{X} \times \mathbf{Y}$. The MI one estimates from the sample is highly dependent on the assumptions about the underlying joint distribution $p(x, y)$; effectively, one estimates MI by estimating the density $p(x, y)$. The most popular method for estimating MI is by using the class of non-parametric estimators built on the method derived by Kraskov et al. [8] (KSG). The KSG estimator uses local geometric information about the sample to approximate the density $p\left(x_{i}, y_{i}\right)$ at each point $\left(x_{i}, y_{i}\right)$ and then calculates a local estimate of MI from it. While this approach has been very successful, there are some weaknesses which we will discuss in this paper. Specifically, the use of local geometric information causes the estimator to not be coordinate invariant in general, which is a violation of the 
basic properties of MI. What's worse, is it's inability to see through useless information, i.e., noise. This is also a consequence of using local information without regard to the overall global structure of the space. When combined, these two problems cause unwanted behavior in even the simplest of situations.

There have been some improvements to KSG [9] which we will discuss. Most often studies of estimators are concerned with its effectiveness with small numbers of samples in large dimension, while here we will be mostly concerned with its robustness under coordinate transformations, redundancy and noise. We will define redundancy and noise more precisely in a later section, but one can also check [7] for a more rigorous definition. As was shown in [1] KSG handles redundant information well, which we will reiterate in a later section. It is KSG's inability to handle noise that diminishes it's effectiveness in real data sets. In the next section we will briefly discuss the basic properties of MI, and then discuss the ideas behind non-parametric entropy estimators. We will then examine the robustness of KSG and it's improvements in section IV. We end with a discussion.

\section{Mutual Information}

We will reiterate some the basic properties of mutual information. Consider two spaces of propositions, $\mathbf{X}$ and $\mathbf{Y}$, whose joint space is given by $\mathbf{X} \times \mathbf{Y}$ (The spaces $\mathbf{X}, \mathbf{Y}$ can be either discrete/categorical or continuous.). The global correlations present between the two spaces is determined from the mutual information,

$$
I[\mathbf{X} ; \mathbf{Y}]=\int d x d y p(x, y) \log \frac{p(x, y)}{p(x) p(y)}
$$

where $p(x, y)$ is the joint probability density and,

$$
p(x)=\int d y p(x, y)=\int d y p(y) p(x \mid y) \quad \text { and } \quad p(y)=\int d x p(x, y)=\int d x p(x) p(y \mid x)
$$

are the marginals. The product marginal $p(x) p(y)$ can be interpreted as an independent prior and the MI gives a ranking of joint distributions $p(x, y)$ according to their amount of correlation; joint distributions with more correlation have higher values of MI. The MI is bounded from below by $I_{\min }=0$ whenever the spaces $\mathbf{X}$ and $\mathbf{Y}$ are uncorrelated; i.e., $p(x, y)=p(x) p(y)$, and is typically unbounded from above (except in cases of discrete distributions).

One immediate consequence of the functional form of (1) is its invariance under coordinate transformations. Since the probabilities,

$$
p(x, y) d x d y=p\left(x^{\prime}, y^{\prime}\right) d x^{\prime} d y^{\prime}
$$

are equivalent, then $I[\mathbf{X} ; \mathbf{Y}]=I\left[\mathbf{X}^{\prime} ; \mathbf{Y}^{\prime}\right]$. While this fact is somewhat trivial on its own, when combined with other types of transformations it can be quite powerful. In this paper we will study three main types of transformations, the first being coordinate transformations. The second kind of transformation of interest is marginalization, and the third is products. Marginalization is simply the the projecting out of some variables, which according to the design criteria of MI [7], can only ever decrease the correlations present. On the other hand, products of spaces can increase correlations when the new variables provide new information. The most trivial type of product is an embedding.

One advantage of $\mathrm{MI}$ is its invariance under the inclusion of redundant information. For example, consider adding to the space $\mathbf{X}$ another space which is simply a function of $\mathbf{X}$, i.e., $\mathbf{X} \rightarrow \mathbf{X} \times f(\mathbf{X})$. The joint probability distribution becomes,

$$
p(x, f(x), y)=p(x, f(x)) p(y \mid x, f(x))=p(x) \delta(f(x)-f) p(y \mid x)
$$


and hence the MI is,

$$
I[\mathbf{X} \times f(\mathbf{X}) ; \mathbf{Y}]=\int d x d y d f p(x) \delta(f(x)-f) p(y \mid x) \log \frac{p(x) p(y \mid x)}{p(x) p(y)}=I[\mathbf{X} ; \mathbf{Y}]
$$

The map $\mathbf{X} \rightarrow \mathbf{X} \times f(\mathbf{X})$ is an embedding of $\mathbf{X}$ into a higher dimensional space. Such a transformation does not increase the intrinsic dimension of $\mathbf{X}$. Machine learning algorithms exploit this type of transformation in conjunction with coordinate transformations and marginalizations.

Much like in Equation (4), MI is also invariant under the addition of noise, which are defined as variables, $\mathbf{Z}$, that are uncorrelated to both $\mathbf{X}$ and $\mathbf{Y}$,

$$
p(x, z, y)=p(x, z) p(y \mid x, z)=p(x) p(z) p(y \mid x)=p(z) p(x, y)
$$

And like (5) the mutual information is invariant,

$$
I[\mathbf{X} \times \mathbf{Z} ; \mathbf{Y}]=I[\mathbf{X} ; \mathbf{Y} \times \mathbf{Z}]=\int d x d y d z p(z) p(x, y) \log \frac{p(x, y)}{p(x) p(y)}=I[\mathbf{X} ; \mathbf{Y}]
$$

Unlike with redundancy, noise variables necessarily increase the dimension of the underlying space.

\section{Non-Parametric Estimation}

Non parametric entropy estimators attempt to utilize the geometry of the underlying sample to estimate the local density and hence the local entropy. A popular estimator is the one developed by Kozachenko and Leonenko (KL) [10], which we will briefly motivate. Consider the task of estimating the entropy of a sample $\mathcal{X}$ from an underlying space $\mathbf{X}$. Our goal is to find an unbiased estimator of the form $\hat{H}[\mathcal{X}]=N^{-1} \sum_{i=1}^{N} \log p\left(x_{i}\right)$, which converges to the true Shannon entropy as $N \rightarrow \infty$ (We highlight the word true here, since the underlying probability distribution is not known and hence our estimation depends on our assumptions about its form.). To find an approximation of $\log p\left(x_{i}\right)$, consider the following probability distribution,

$$
P_{\varepsilon}\left(x_{i}\right) d \varepsilon=\frac{\left(N_{1}\right) !}{1 !(k-1) !(N-k-1) !} p_{i}^{k-1}\left(1-p_{i}\right)^{N-k-1} \frac{d p_{i}}{d \varepsilon} d \varepsilon
$$

which is the probability that the $k$ th-nearest neighbor of the point $x_{i}$ exists within the small spherical shell of radius $\varepsilon / 2$ and that there are $k-1$ points at $r_{i}<\varepsilon / 2$ and $N-k-1$ points at $r_{i}>\varepsilon / 2+d \varepsilon$. This distribution is of course properly normalized, and upon evaluating the expected value of the logarithm of $p_{i}$, we find,

$$
\left\langle\log p_{i}\right\rangle=\int d \varepsilon P_{\varepsilon}\left(x_{i}\right) \log p_{i}=\psi(k)-\psi(N)
$$

where $\psi(k)$ is the digamma function. From here one can determine an approximation for the logarithm of the true distribution by assuming something about the local behavior of $p\left(x_{i}\right)$ with respect to the probability mass $p_{i}$. In the KL approximation (and as well in the KSG approximation), it is assumed that the probability within the region defined by $p_{i}$ is uniform with respect to the true distribution at the point $x_{i}$

$$
p_{i} \approx c_{d} \varepsilon^{d} p\left(x_{i}\right)
$$

where $d$ is the dimension of the space and $c_{d}$ is the volume of the unit $d$-ball (The form of $c_{d}$ depends on the choice of metric for the space $\mathbf{X}$. As we will see a useful choice for MI estimation is the $L^{\infty}$ norm.). Putting (10) into the unbiased estimator one arrives at,

$$
\hat{H}[\mathcal{X}]=\psi(N)-\psi(k)+\log c_{d}+\frac{d}{N} \sum_{i=1}^{N} \log \left(\varepsilon_{i}\right)
$$




\subsection{The Vanilla KSG Estimator}

The KSG estimator of the first kind is derived by taking the expression in (11) and applying it to the decomposition,

$$
\hat{I}[\mathcal{X} ; \mathcal{Y}]=\hat{H}[\mathcal{X}]+\hat{H}[\mathcal{Y}]-\hat{H}[\mathcal{X}, \mathcal{Y}]
$$

where $\hat{H}[\mathcal{X}, \mathcal{Y}]$ is the entropy over the joint distribution $p(x, y)$. As has been shown and argued by KSG, the approximation above is slightly naive since the local densities in the joint and marginal spaces can be different, leading to errors in the terms involving $\log \left(\varepsilon_{i}\right)$ which don't necessarily cancel. As a neat trick, KSG suggests using the same density found in the joint space in the marginal spaces, so that the factors $\left(d_{x} / N\right) \sum_{i=1}^{N} \log \left(\varepsilon_{i}^{x}\right),\left(d_{y} / N\right) \sum_{i=1}^{N} \log \left(\varepsilon_{i}^{y}\right)$ and $\left(\left(d_{x}+d_{y}\right) / N\right) \sum_{i=1}^{N} \log \left(\varepsilon_{i}^{x y}\right)$ will cancel. Choosing the same density for fixed $k$ in the joint space causes the $k$ values in the marginal spaces to vary, and hence we arrive at the expression,

$$
\hat{I}^{1}[\mathcal{X} ; \mathcal{Y}]=\psi(k)+\psi(N)-\left\langle\psi\left(n_{x}+1\right)\right\rangle-\left\langle\psi\left(n_{y}+1\right)\right\rangle
$$

where $n_{x}$ and $n_{y}$ are the number of points which land in the $d_{x}$ and $d_{y}$ balls of radius $\varepsilon / 2$ in the marginal spaces.

One unfortunate consequence of the KSG estimator of the first kind is its reliance on the $L^{\infty}$ norm for finding neighbors. As has been pointed out by others[9], such a choice can lead to problems in regions where the probability varies greatly, which can easily happen in spaces of large dimension. Unless the density of samples increases exponentially with respect to the dimension of the space, the errors in choosing $L^{\infty}$ will compound quickly.

\subsection{The LNC Correction to KSG}

As an attempted correction to KSG's problem with using the $L^{\infty}$ box, S. Gao et al. proposed the local non-uniform correction (LNC) technique. This technique adjusts the unbiased estimator for MI by replacing the $L^{\infty}$ volume in the joint space with a volume computed from a PCA analysis. The basic idea is the following. Consider a point $x_{i}$ whose $k$ th-neighbor is $x_{k}$. With the collection of $k+1$ points including $x_{i}, x_{k}$ and all points closer than $x_{k}$, construct the correlation matrix $C_{i j}$ and find its eigenvectors. By then projecting each point along the maximal eigenvectors, we can find a PCA bounding box, which is rotated and skewed with respect to the $L^{\infty}$ box. The assumption in this case is that the rotated PCA box is a much better representation of the region of uniform probability around $x_{i}$. Once each volume is found, the MI is given by,

$$
\hat{I}_{L N C}=\hat{I}_{K S G}-\frac{1}{N} \sum_{i=1}^{N} \log \frac{\bar{V}_{i}}{V_{i}}
$$

where $\bar{V}_{i}$ is the PCA volume and $V_{i}$ is the $L^{\infty}$ volume. Such an estimator has shown to give vast improvement to the naive KSG method, however current results are limited to two dimensional problems. The reason for this is its inability to deal with redundant information. To see this, consider a two-dimensional problem in which the variables $\mathbf{X} \times \mathbf{Y}$ have some non-trivial correlations. If we add to $\mathbf{X}$ a redundant copy, $\mathbf{X} \rightarrow \mathbf{X} \times f(\mathbf{X})$, then we expect the MI to be invariant. If one naively uses the LNC method, one will find that the MI increases. This is because the volumes $\bar{V}_{i}$ will most often decrease when computed in the redundant scenario and hence the LNC correction term will most often increase.

A possible fix to this problem is to not only correct the volume in the joint space, but to fix the volumes in the marginal spaces as well, leading to the LNC correction of the second kind,

$$
\hat{I}_{L N C^{2}}=\hat{I}_{K S G}-\frac{1}{N} \sum_{i=1}^{N} \log \frac{\bar{V}_{i}^{x y}}{\bar{V}_{i}^{x} \bar{V}_{i}^{y}}
$$


In investigating the efficacy of such a method, we discovered that it's not very robust. This is mainly due to the fact that like the original method in (14), (15) is not coordinate invariant in general, and while the volume supplied by redundant variables can in principle be canceled in the denominator of (15), the volumes themselves will be computed with respect to spaces of different dimension, and will therefore not exactly cancel. The effect is to still increase MI under the influence of redundant variables, which is undesirable since vanilla KSG is most successful in this domain. Thus while LNC fixes one aspect of KSG, it reduces its efficacy in another aspect.

\section{Robustness Tests of NP Estimators}

We will access the robustness of the KSG estimator and its variants with respect to the three types of transformations outlined in section two (coordinate transformations, redundancy and noise). Most tests in this section will use a multivariate normal distribution, $\mathcal{N}_{k}=$ $\left((2 \pi)^{k}|\boldsymbol{\Sigma}|\right)^{-1 / 2} \exp \left[-\frac{1}{2}(\mathbf{x}-\mu)^{T} \boldsymbol{\Sigma}^{-1}(\mathbf{x}-\mu)\right]$ where $\boldsymbol{\Sigma}$ is the covariance matrix. The mutual information between two sets of variables $\mathbf{X}_{n}$ and $\mathbf{X}_{m}$, where $n+m=k$, is given by,

$$
I_{\mathcal{N}_{k}}\left[\mathbf{X}_{n} ; \mathbf{X}_{m}\right]=-\frac{1}{2} \log \left(\frac{\left|\Sigma_{k}\right|}{\left|\Sigma_{n}\right||\Sigma|_{m} \mid}\right)
$$

where $\left|\Sigma_{k}\right|$ is the determinant of the covariance matrix $\Sigma_{k}$. For computing the KSG estimate, we will use a python package called (NPEET) [11] developed by G. ver Steeg et al. For the LNC correction we use a similar package [9].

\subsection{Coordinate Transformations}

Since KSG uses the $L^{\infty}$ norm to define the region of uniform probability for the estimate of $p_{i}$, this automatically presents a problem with coordinate invariance. KSG will not even be invariant under linear scalings of the data, let alone arbitrary coordinate transformations (This is likely the motivation for KSG's estimator of the second kind, which gave different weight to each of the variables, however they were unable to derive a closed form expression.). Essentially, if one variable, say $z$, is scaled by a large order of magnitude with respect to the other variables, then the side lengths of the $L^{\infty}$ box will get chosen to be the length of the $k$ th nearest neighbor in the direction of $z$. While this will not necessarily cause a problem with the values of $\left\langle\psi\left(x_{z}+1\right)\right\rangle$, it will cause the counts for the other variables to be much larger than they necessarily should be. As an example, consider the following bivariate normal case.

As one can see from the figure, scaling one variable of the bivariate normal by $10^{5}$ renders the KSG method useless. While one can always argue for a heuristic scaling method, any robust method for computing MI should be invariant under arbitrary scalings. Below is the same experiment using the LNC method.

While the LNC method corrects the behavior of the curve in the highly correlated region, it still fails to capture the correct value overall due to KSG's inability to handle arbitrary coordinate transformations. The effects of arbitrary coordinate transformations are even worse in higher dimensional situations. Consider the eight-dimensional multivariate Gaussian below.

As you can see, multiplying all the variables by a factor of 10 greatly reduces the accuracy of the KSG algorithm. To see this effect happening more gradually, we focus on one particular value of the correlation matrix (where all correlation coefficients are equal to 1/2) and dial up the transformation on the four variables in one set. The results for 10,000 and 100,000 points are below.

As you can see, the KSG approximation begins to deteriorate very quickly under linear transformations when the dimensionality is high. Increasing the number of points by a factor of ten seems to do little to help this. While this is certainly a flaw in the method, it isn't as dire as the others we will explore in the next section. For now, one can adopt a strategy in which each variable is scaled in a way that gives equal weight to each of them. Proposed methods for this were given in [1]. 


\subsection{Redundancy vs. Noise}

Another simple test we can perform is to see how MI in high dimensional situations handles redundant and noisy variables. Specifically, we will look at how the MI changes as we dial up the noise present in redundant variables by randomizing their values with respect to the other variables. As we saw in [1], KSG does quite well with redundant variables, however noisy variables still present a problem.

We have studied the ability of vanilla KSG to calculate MI under the presence of redundant variables in [1]. Here we will briefly discuss the highlights. We examined a binary decision problem in which two distributions (signal and background) are separated by a certain amount with respect to their means. We showed in [1] that the neural network transformation leaves the MI unchanged which is expected according to its design criteria [7]. We tested this claim on a more general data set which was generated as part of a machine learning analysis on a mock SUSY search [12]. The SUSY data set contains eight low-level variables and ten high-level variables which are functions of the low-level ones. From the tests in [1], we again see that KSG performs well under the addition of the high-level variables when they are redundant.

However when the variables are shuffled so that they are independent of both $\mathbf{X}$ and $\boldsymbol{\Theta}$, i.e., when they are noise, then KSG starts to deteriorate very quickly. We can see this effect in the case where the $\mathrm{MI}$ is known exactly. Using the multi-variate Gaussian with eight variables from the previous tests, we added three copies of the last variable on one side, essentially taking $x_{4} \in X$ and creating $X^{\prime}=X \cup x_{4} \cup x_{4} \cup x_{4}$. We then dialed up the randomness in the three variables to $100 \%$. What we mean here is that we rearranged the points in the set $\left(x_{4} \cup x_{4} \cup x_{4}\right)$ so that a particular value $x_{i} \in\left(x_{4} \cup x_{4} \cup x_{4}\right) \neq f(\mathbf{X})$ and $x_{i} \in\left(x_{4} \cup x_{4} \cup x_{4}\right) \neq f(\mathbf{Y})$.

This shows that noisy variables cause the KSG estimate to go down as a function of their uselessness. This problem compounds quickly when the number of useless variables increases, making KSG's ability to determine MI in high-dimensional cases problematic.

\section{Discussion}

We've shown in practical examples that KSG is not robust under coordinate transformations (Figures 1-4) and noise (Figures 5 and 6). While the effect of including noise is not as drastic in cases of simple distributions (Figure 3), it is must more dramatic in cases where the the distribution is not simple (Figure 5).
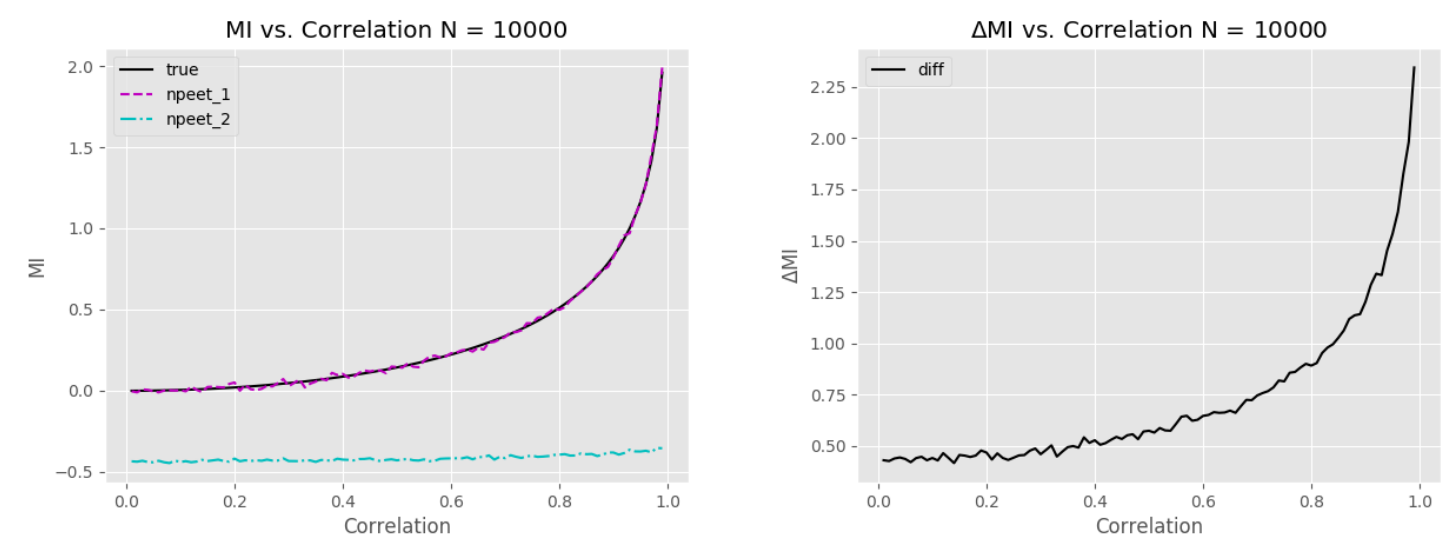

Figure 1. Comparison of mutual information estimates KSG for a bivariate normal distribution before (npeet_1) and after (npeet_2) a linear transformation of one variable by a factor of $10^{5}$. The second plot shows the difference between (npeet_1) and (npeet_2). 

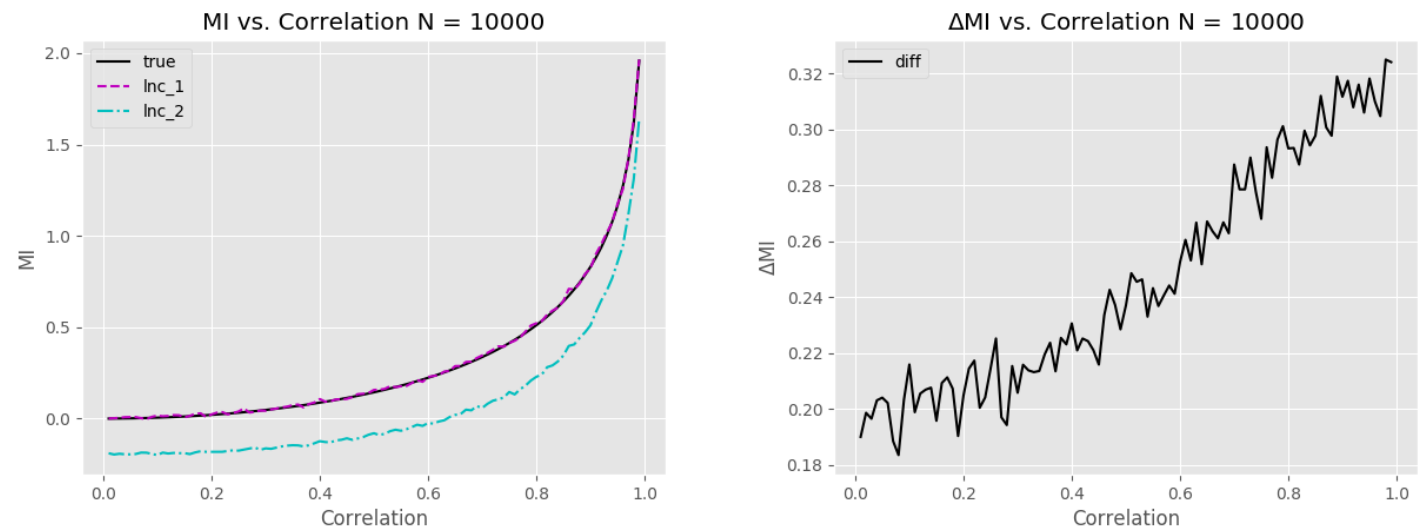

Figure 2. Comparison of mutual information estimates LNC for a bivariate normal distribution before (lnc_1) and after (lnc_2) a linear transformation of one variable by a factor of $10^{5}$. The second plot shows the difference between (lnc_1) and (lnc_2).
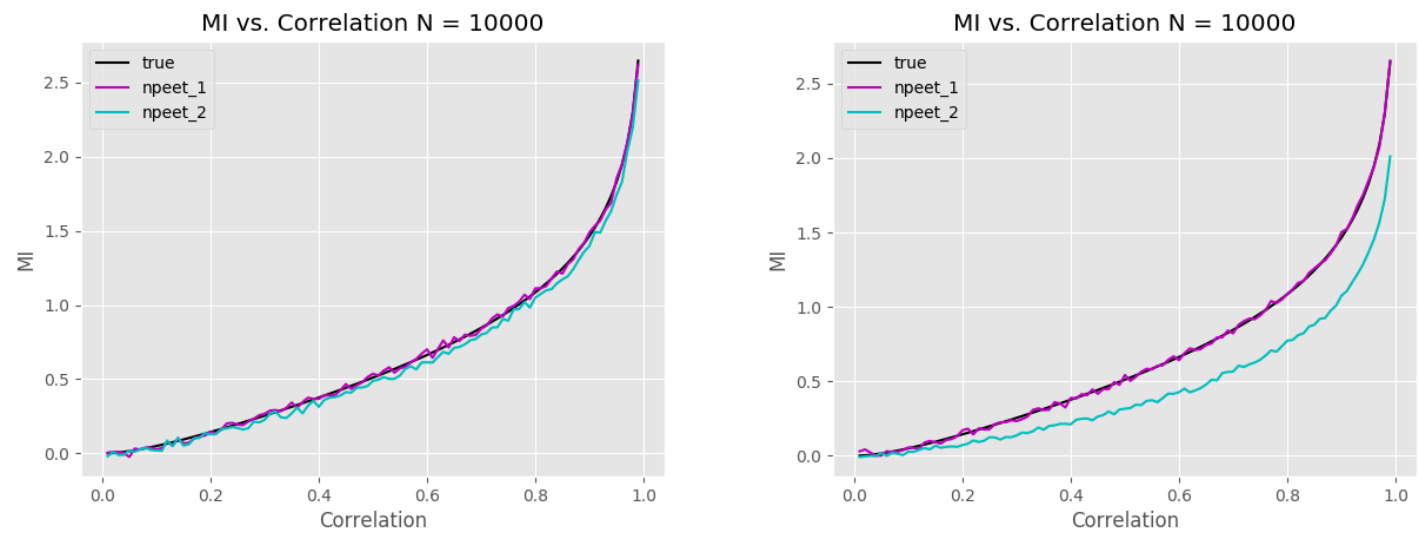

Figure 3. Comparison of mutual information estimates KSG for a multivariate normal distribution with equal correlation coefficients $\rho_{i j}$ before (npeet_1) and after (npeet_2) a linear transformation of one variable by a factor of 10 . The second plot compares the KSG estimators after four variables are multiplied by a factor of 10 .
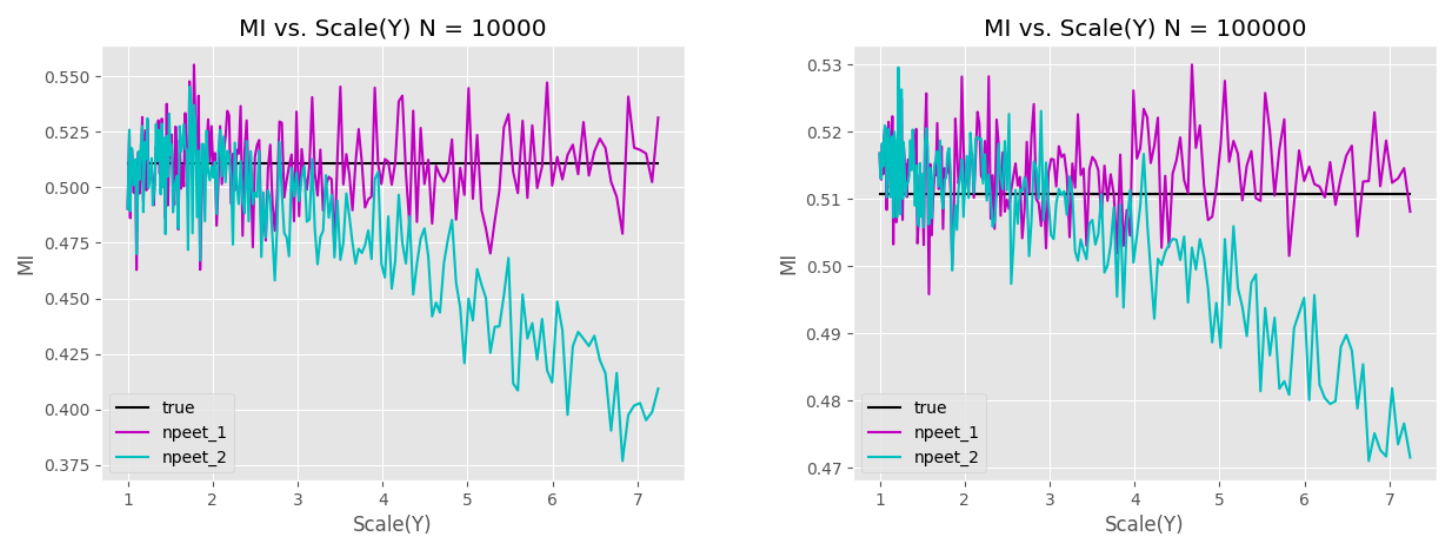

Figure 4. Comparison of true mutual information between the eight variables of a multivariate normal distribution where all correlation coefficients are equal to $1 / 2$ (black line) and the value estimated from $N=10,000$ and $N=100,000$ samples before (npeet_1) and after (npeet_2) a linear transformation is applied to one variable $x \rightarrow x^{\prime}=10 x$. 

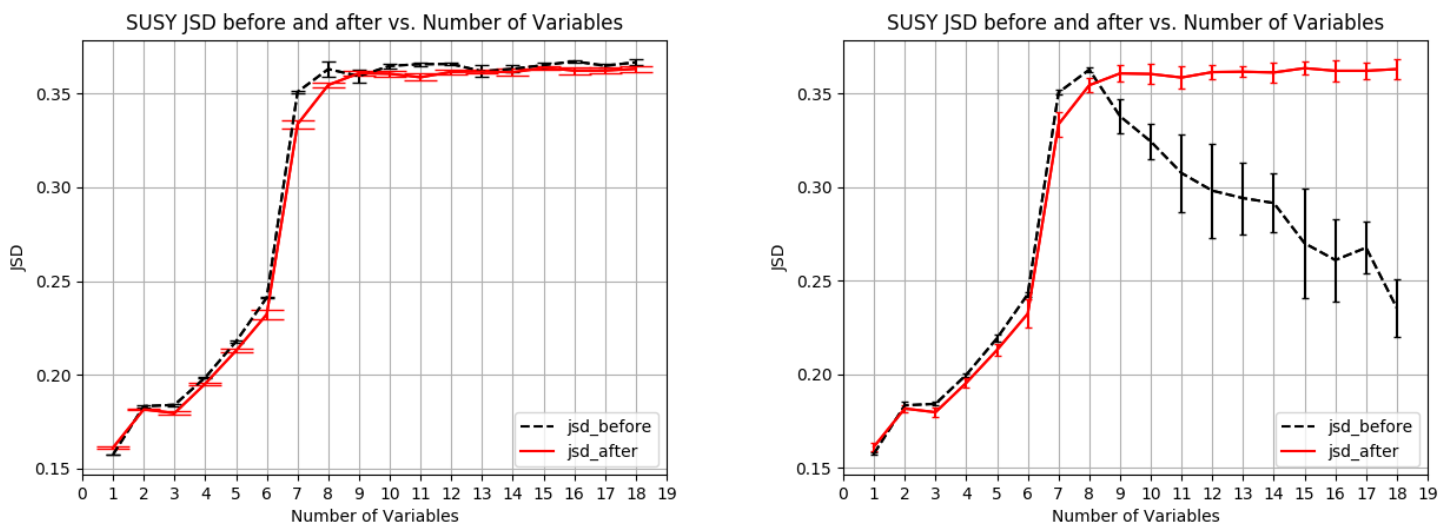

Figure 5. Comparison of MI values for increasing additions of discriminating variables for the SUSY data set. The first eight variables are low-level and the last ten are functions of the first eight. The first plot shows a comparison of (NN) performance when the last ten variables are redundant while the second shows how KSG's accuracy deteriorates when the high-level variables are shuffled.
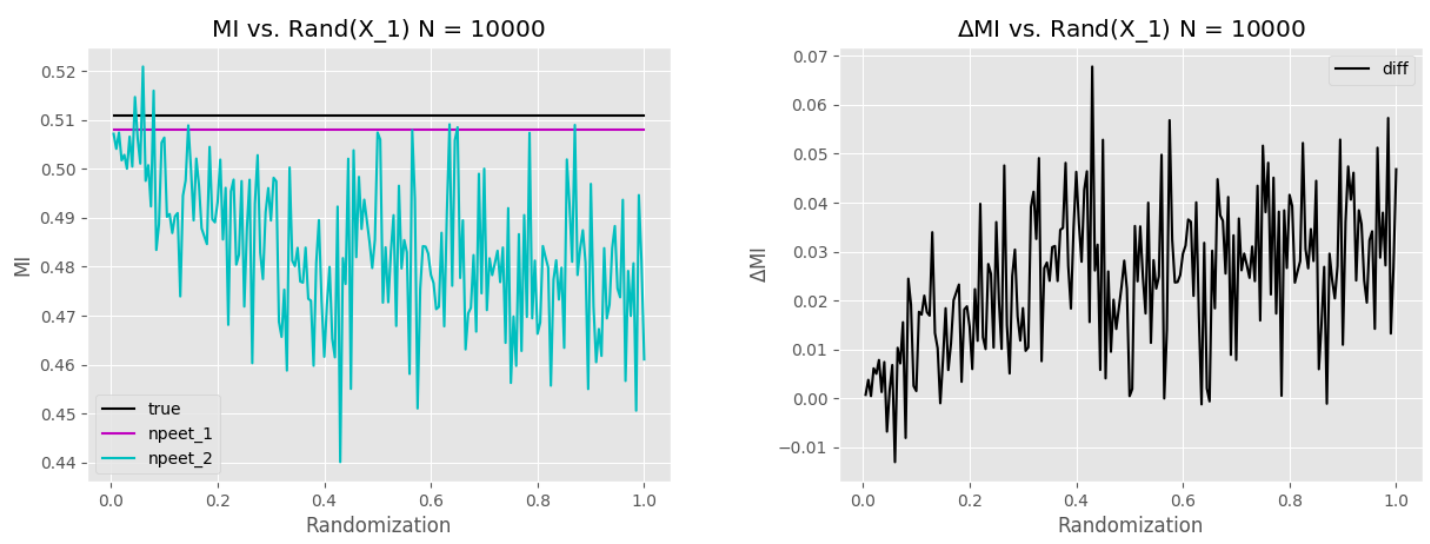

Figure 6. Comparison of true mutual information between the eight variables of a multivariate normal distribution where all correlation coefficients are equal to $1 / 2$ (black line) and the value estimated from $N=10,000$ samples before (npeet_1) and after (npeet_2) a randomization is applied to the set of redundant variables.

Acknowledgments: We would like to thank A. Caticha, K. Knuth, G. ver Steeg and K. Vanslette for insightful conversations.

\section{References}

1. Carrara, N.; Ernst, J.A. On the Upper Limit of Separability. arXiv 2017, arXiv:1708.09449.

2. Tishby, N.; Pereira, F.C.; Bialek, W. The information bottleneck method. arXiv 2000, arXiv:physics/0004057.

3. Tishby, N.; Zaslavsky, N. Deep Learning and the Information Bottleneck Principle. arXiv 2015, arXiv:1503.02406.

4. Chen, X.; Duan, Y.; Houthooft, R.; Schulman, J.; Sutskever, I.; Abbeel, P. InfoGAN: Interpretable Representation Learning by Information Maximizing Generative Adversarial Nets. arXiv 2016, arXiv:1606.03657.

5. Ver Steeg, G.; Galstyan, A. The Information Sieve. arXiv 2015, arXiv:1507.02284.

6. Cover, T.M.; Thomas, J.A. Elements of Information Theory; Wiley-Interscience: Hoboken, NJ, USA, 2006.

7. Carrara, N.; Vanslette, K. The Inferential Foundations of Mutual Information. arXiv 2019, arXiv:1907.06992.

8. Kraskov, A.; Stögbauer, H.; Grassberger, P. Estimating mutual information Phys. Rev. E 2004, 69, 066138.

9. Gao, S.; Ver Steeg, G.; Galstyan, A. Efficient Estimation of Mutual Information for Strongly Dependent Variables. arXiv 2014, arXiv:1411.2003. 
10. Kozachenko, L.F.; Leonenko, N.N. A statistical estimate for the entropy of a random vector. Probl. PeredachiInformats 1987, 2, 9-16.

11. Ver Steeg, G. Non-parametric Entropy Estimation Toolbox. Available online: https://github.com/ gregversteeg/NPEET (accessed on 14 January 2020).

12. Baldi, P.; Sadowski, P.; Whiteson, D. Searching for exotic particles in high-energy physics with deep learning. Nat. Commun. 2014, 5, 4308.

(C) 2020 by the authors. Licensee MDPI, Basel, Switzerland. This article is an open access article distributed under the terms and conditions of the Creative Commons Attribution (CC BY) license (http://creativecommons.org/licenses/by/4.0/). 